\title{
Nebenwirkungen untergraben Blutdrucktherapie
}

\author{
Hypertoniker bewerten die unerwünschten Nebenwirkungen von Blutdrucksenkern unterschiedlich. \\ Manche haben deutlich stärkere Auswirkungen auf die Therapietreue. Nachfragen lohnt sich!
}

\begin{abstract}
Für eine interessante Studie beobachteten die Autoren 175 Hypertoniker ohne nennenswerte Begleiterkrankungen, die erstmals oder nach einer Unterbrechung von mindestens zwei Monaten eine antihypertensive Therapie begannen. Sie sammelten nach 3, 6, 9 und 12 Monaten Daten über Zahl, Art und Dosis der Antihypertensiva, befragten die Patienten systematisch nach Nebenwirkungen und überprüften die Tabletteneinnahme.
\end{abstract}

Bei $85 \%$ der Patienten trat mindestens eine Nebenwirkung auf, die mittlere Häufigkeit lag bei vier. 34,5\% der $\mathrm{Pa}$ tienten nahmen weniger als $80 \%$ der verordneten Tabletten ein, was als unzureichende Adhärenz definiert wurde. Die mangelhafte Therapietreue nahm mit der Zahl der Nebenwirkungen zu, nicht aber mit ihrem Schweregrad.

Nebenwirkungen des Urogenitaltrakts - beispielsweise Harndrang tagsüber und in der Nacht, Erektionsstörungen und Libidoverlust - beeinträchtigten die Tabletteneinnahme deutlich und signifikant stärker als andere organbezogene Beschwerden. Dazu zählten neben kardiopulmonalen und gastrointestinalen auch anticholinerge Effekte wie ein trockener Mund oder Sehstörungen, ferner funktionelle Probleme wie Müdigkeit und Schwäche, oder auch neuropsychiatrische Effekte wie Schlaflosigkeit oder depressive Verstimmungen.

- Tedla YG, Bautista LE. Drug side effect symptoms and adherence to antihypertensive medication. Am J Hypertens. 2016;29:772-9

\begin{abstract}
KOMMENTAR
Mangelhafte Therapietreue - auf Befragen

zugegeben oder verschwiegen - ist ein häufiges Problem bei der Hypertonie und anderen Krankheiten, solange sie noch keine Beschwerden verursachen. Als häufige Ursachen dafür gelten Nebenwirkungen.

Die erwünschten Wirkungen der Arzneimittel wurden als Voraussetzung für die Zulassung schon immer intensiv erforscht, doch mit den Schattenseiten der Pharmaforschung verdient man keine Lorbeeren. Angesichts dieser Datenlage gibt die vorliegende Studie einen kleinen, aber wichtigen Hinweis. Besonders Nebenwirkungen aus dem sensiblen Bereich des Urogenitaltrakts beeinträchtigen die Compliance und sollten deshalb vom Therapeuten angesprochen werden.
\end{abstract}

Prof. Dr. med. H. Holzgreve

\section{Blinder Passagier aus Nigeria}

Nach einem Urlaub in Nigeria waren bei einem 42-jährigen Mann heftig juckende Effloreszenzen am rechten Fuß aufgetreten. Die rötlichen, gewundenen Stränge veränderten sich täglich und wanderten teils zentimeterweit. Bei der Untersuchung zeigten sich geschlängelte, bandförmige erythematöse Effloreszenzen an der Außenseite des rechten Fußes wie sie typisch für eine Larva migrans sind. Der Patient wurde systemisch mit Albendazol ( $800 \mathrm{mg} / \mathrm{d}$ oral für 3 Tage) behandelt, worunter die Effloreszenzen innerhalb von zwei Wochen vollständig abheilten. Auch zwei Monate später war es nicht zu einem Rezidiv gekommen.

Die Larva migrans wird durch Hautkontakt mit den Larven des Hakenwurms erworben, etwa beim Barfußlaufen oder beim Liegen am Strand. Der Parasit kommen in den Faeces infizierter Hunde und Katzen in tropischen Ländern vor und bohrt sich durch die menschliche Haut. Er kann sich nur innerhalb der Epidermis bewegen, da er keine Kollagennase besitzt, um die Basalmembran zu penetrieren. Die juckenden Effloreszenzen sind Ausdruck einer Immunreaktion auf die Larven und ihre Produkte. Die stark juckenden und gewundenen Gänge an Füßen, Rücken, Gesäß, Oberschenkeln und Abdomen sind so charakteristisch, dass Differenzialdiagnosen kaum in Betracht kommen.

Die Erkrankung ist zwar selbstlimitierend, sollte aber wegen des ausgeprägten und anhaltenden Juckreizes und der Gefahr einer möglichen Superinfektion durch Kratzexkoriationen, aber auch allgemein wegen der psychischen Belastung eines Parasitenbefalls medikamentös behandelt
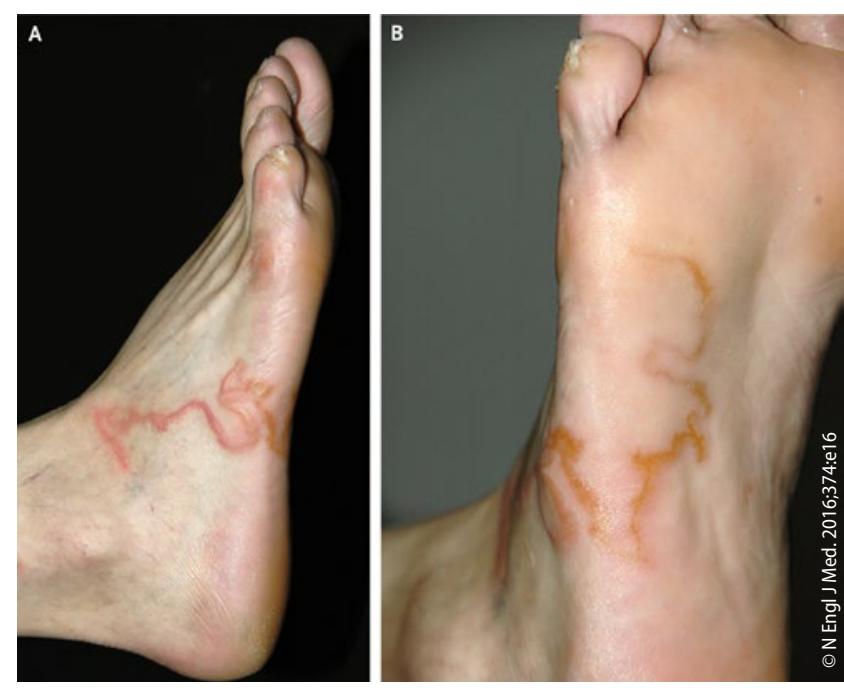

A, B: Geschlängelte, bandförmige erythematöse Effloreszenzen.

werden. Bei Vorbehalten gegen eine systemische Therapie kommt auch eine topische Behandlung mit 10\%-Albendazol-Creme infrage.

Prof. Dr. med. H. S. FüeßI

- Ma DL, Vano-Galvan S (mdonglai@sohu.com). Creeping eruption - cutaneous larva migrans. NEngl J Med. 2016;374:e16 